\title{
Diabetologia intervencional: uma nova abordagem para o tratamento cirúrgico do diabetes tipo 2
}

Bruno Geloneze'

$\mathrm{O}$ manejo clínico de pacientes com obesidade e diabetes sempre constituiu um desafio para a endocrinologia. A unificação do tratamento glucocêntrico, que busca priorizar o controle glicêmico, com um tratamento focado na redução do peso, sempre foi dificultado pela persistência de hábitos alimentares inadequados por parte dos pacientes e consequente potencial ganho de peso ao longo do tratamento.

A cirurgia bariátrica pode ser considerada como um dos maiores e mais contundentes avanços no tratamento do diabetes desde a descoberta da insulina. Nos últimos 15 anos, desde o primeiro estudo observacional, vários estudos prospectivos têm confirmado o benefício da cirurgia bariátrica, em especial nos pacientes portadores de diabetes tipo 2 ( 1 ).

Várias entidades médicas e serviços públicos de saúde mundiais recomendam a cirurgia bariátrica como uma opção para o tratamento de adultos com índice de massa corporal (IMC) $\geq 35 \mathrm{~kg} / \mathrm{m}^{2}$ e comorbidades com potencial de melhora clínica significativa, destacando-se o diabetes tipo 2 (2).

Os mecanismos de melhora do diabetes com as cirurgias consagradas (bypass gástrico com reconstrução em Y de Roux, ou como conhecida no Brasil, cirurgia de Fobi-Capella e derivação biliopancreática, ou cirurgia de Scopinaro) são apenas parcialmente conhecidos. Sabemos que ocorrem melhora da resistência à insulina, redução da adiposidade e adiposopatia, aumento de GLP-1 e do efeito incretina per se, culminando com uma melhora da função da célula beta-pancreática. Novos mecanismos vêm sendo descritos, tais quais modulação da neoglicogênese intestinal, mudança na microbiota, efeitos sistêmicos dos ácidos biliares, redução da inflamação hipotalâmica, entre outros $(3,4)$. Estamos diante da nova era da terapêutica baseada na mudança anatômica e funcional deste importante órgão endócrino e metabólico: o tubo digestivo.

Os efeitos da cirurgia no controle do diabetes são contundentes, porém a validade dos dados disponíveis é questionável. Falhas metodológicas existem resultantes das altas taxas de perda de seguimento na maioria dos estudos, além da falta de grupos controle adequados e da curta duração destes (5). A curta duração dos estudos (em geral menor que 2 anos) é adequada aos propósitos de pesquisa em cirurgia, visto que a maioria das complicações cirúrgicas ocorre nesse período. A cirurgia bariátrica pode acarretar efeitos colaterais por toda a vida, como deficiências nutricionais e osteoporose, que podem aparecer e se agravar após anos de seguimento (6). Além disso, o

${ }^{1}$ Membro do International Diabetes Task Force for Interventional Diabetology do International Diabetes Federation (IDF).

Coordenador do Laboratório de Investigação em Metabolismo e Diabetes (Limed), Universidade Estadual de Campinas (Unicamp), Campinas, SP, Brasil

Correspondência para: Bruno Geloneze

Laboratório de Investigação em Metabolismo e Diabetes (Limed), Unicamp

Rua Carlos Chagas, 420,

Cidade Universitária - Barão Geraldo 13081-970 - Campinas, SP, Brasil bgeloneze@terra.com.br

Recebido em 12/Jul/2011 Aceito em 7/Ago/2011 
diabetes é uma doença crônica, e expressões como cura, remissão e melhora da doença devem ser utilizadas com maior prudência.

Equipes multidisciplinares são necessárias para a assistência aos candidatos à cirurgia. A exigência da existência de uma equipe já existe no Brasil, porém somente em 2010 o Conselho Federal de Medicina lançou uma nova resolução que incluiu o Endocrinologista como parte dessa equipe. Talvez isso seja reflexo da necessária integração entre as áreas clínicas e cirúrgicas pelo bem dos pacientes.

O presente documento publicado na edição de agosto de 2011 nos $A B E \mathcal{O} M$ pela International Diabetes Federation (IDF) traz recomendações chave para a incorporação da diabetologia intervencional ao arsenal terapêutico dos Endocrinologistas (7). As principais recomendações do documento são: descrição dos pacientes elegíveis para a cirurgia e quais seriam os prioritários, incorporação da cirurgia bariátrica nos algoritmos de tratamento do diabetes tipo 2 , necessidade de realização de cirurgias apenas em centros de excelência com equipes multidisciplinares com experiência no manejo clínico da obesidade e do diabetes, e desenvolvimento de métodos unificados de registro e divulgação de resultados cirúrgicos.

A IDF apresenta recomendações quanto à busca da excelência na geração de novas evidências científicas. De fato, os estudos de intervenção com novos medicamentos nos trazem lições que devem ser incorporadas na análise crítica dos dados cirúrgicos disponíveis e nos auxiliar no delineamento de novos estudos. A taxa de retenção nos estudos com cirurgia bariátrica, muitas vezes, não ultrapassa os $50 \%$, o que não corresponde a valores aceitáveis em estudos clínicos em diversas áreas da medicina.

$\mathrm{Na}$ pesquisa clínica, novas drogas para o diabetes devem inicialmente ser confrontadas com a metformina, mostrando não inferioridade em termos de eficácia e perfil de segurança. Em termos práticos, se um novo fármaco não atinge esses quesitos, em geral, seu programa de desenvolvimento clínico é abortado. A cirurgia bariátrica, embora contundente, ainda carece de estudos comparando-a a tratamentos clínicos. Logicamente, considerando os riscos cirúrgicos perioperatórios e de desnutrição em longo prazo, a cirurgia deve ser comparada com o melhor tratamento clínico disponível que combine dieta, exercício e múltiplos fármacos.
Novas técnicas cirúrgicas são bem-vindas, porém não podemos recorrer ao empirismo do passado quando a cirurgia bariátrica, "por acaso", mostrou-se eficaz para tratar o diabetes (8). Toda e qualquer nova proposta deve ter um princípio fisiológico bem estudado primeiramente em modelos animais, e muito depois poderá mostrar um perfil de segurança e efetividade que seja comparável às técnicas existentes e bem estudadas em longo prazo, como o bypass gástrico. Após cumprir essas etapas, novas cirurgias podem obter aprovações oficiais e serem consideradas como condutas cirúrgicas alternativas às técnicas já consagradas.

A impressão é que estamos em uma nova era no tratamento do diabético obeso e temos um longo caminho a percorrer para extrair da diabetologia intervencional o melhor para os pacientes. Cuidar dos pacientes com diabetes sempre foi missão dos clínicos, mas cirurgiões têm tido inegável "sucesso" no tratamento do DM2. A aplicação de princípios de conduta ética e científica deverá promover a conciliação dos legítimos interesses tanto de clínicos como de cirurgiões nos cuidados com o paciente com diabetes e obesidade, antes, durante e após a cirurgia. Quem mais lucrará serão os portadores de diabetes.

\section{REFERÊNCIAS}

1. Sjöstrom $L$, Lindroos $A K$, Peltonen $M$, Torgerson J, Bouchard $C$, Carlsson B, et al. Lifestyle, diabetes, and cardiovascular risk factors 10 years after bariatric surgery. N Engl J Med. 2004;351:2683-93.

2. Meijer RI, van Wagensveld BA, Siegert CE, Eringa EC, Serné EH, Smulders YM. Bariatric surgery as a novel treatment for type 2 diabetes mellitus: a systematic review. Arch Surg. 2011;146:744-50.

3. Buchwald H, Estok R, Fahrbach K, Banel D, Jensen MD, Pories WJ, et al. Weight and type 2 diabetes after bariatric surgery: systematic review and meta-analysis. Am J Med. 2009;122(3):248-256.e5.

4. Lima MM, Pareja JC, Alegre SM, Geloneze SR, Kahn SE, Astiarraga $B D$, et al. Acute effect of roux-en-y gastric bypass on whole-body insulin sensitivity: a study with the euglycemic-hyperinsulinemic clamp. J Clin Endocrinol Metab. 2010;95(8):3871-5.

5. van de Sande-Lee S, Pereira FR, Cintra DE, Fernandes PT, Cardoso AR, Garlipp CR, et al. Partial reversibility of hypothalamic dysfunction and changes in brain activity after body mass reduction in obese subjects. Diabetes. 2011;60(6):1699-704.

6. Koch TR, Finelli FC. Postoperative metabolic and nutritional complications of bariatric surgery. Gastroenterol Clin North Am. 2010;39(1):109-24.

7. Dixon JB, Zimmet P, Alberti KG, Rubino F. Bariatric surgery: an IDF statement for obese type 2 diabetes. Diabet Med. 2011;28:628-42.

8. Pories WJ, MacDonald KG Jr, Flickinger EG, Dohm GL, Sinha MK, Barakat HA, et al. Is type II diabetes mellitus (NIDDM) a surgical disease? Ann Surg. 1992;215(6):633-42. 